DOI: https://doi.org/10.11144/Javeriana.upsy20.vccd

\title{
Variabilidad y cambio en la comprensión de deseos en niños preescolares: un estudio microgenético*
}

\section{Variability and Change in Understanding of Desires in Preschoolers: a Microgenetic Study}

\author{
Juan Pablo Arroyave Hurtado ${ }^{a}$ \\ Universidad de Antioquia, Colombia \\ ORCID: https://orcid.org/0000-0002-0582-1495 \\ Johny Villada Zapata \\ Universidad de Antioquia, Colombia \\ ORCID: https://orcid.org/0000-0002-9561-5344 \\ Milton Eduardo Bermudez Jaimes \\ Pontificia Universidad Javeriana, Colombia \\ ORCID: https://orcid.org/0000-0002-5049-7073
}

a Autor de correspondencia. Correo electrónico: jpablo.arroyave@udea.edu.co

Para citar este artículo: Arroyave, J. P., Villada, J., \& Bermúdez, M. (2021). Variabilidad y cambio en la comprensión de deseo en niños preescolares: un estudio microgenético. Universitas Psychologica, 20, 1-14. https://doi.org/10.11144/Javeriana.upsy20.vccd

\section{RESUMEN}

Este estudio analiza la capacidad de 14 niños (35\% varones) entre 2 y 3 años $(M=2.9 ; S D=0.17)$ para comprender los deseos. La literatura ha dado por sentado que niños de esas edades comprenden deseos simples, amparándose en una postura estructuralista del desarrollo, en la que básicamente hay una descripción de hitos que están relacionados con la edad. En contraposición a esta tradición, este estudio busca dar cuenta del cambio y la variabilidad, apoyándose en la teoría de los Sistemas Dinámicos No Lineales. Por ello, usando el método microgenético, se evaluó durante varias sesiones la capacidad de los niños para usar los deseos de otros para predecir emociones y comportamientos. Se adaptaron las tareas clásicas de Wellman y Wooley (1990), y se les presentaron a los niños en 5 sesiones, en las que había 3 variaciones de las tareas. Los datos se analizaron con la técnica de mínimos y máximos, y se encontraron un conjunto de trayectorias con muchas fluctuaciones, que distan de mostrar desempeños altos y estables como lo afirman estudios previos. Se concluye que en la investigación sobre el desarrollo, las visiones acumulativas y homogéneas dificultan la observación del cambio y de la amplitud del mismo, por ello se requiere hacer hincapié en la variabilidad.

Palabras clave

deseos; teoría de la mente; desarrollo; variabilidad; sistemas dinámicos; niños preescolares.

\begin{abstract}
This study analyzes the ability of 14 children (65\% girls) between 2 and 3 years old $(M=2.8 ; S D=0.17)$ to understand wishes. The literature has taken for granted that children of these ages understand simple desires, relying on a structuralist posture of development, where there is basically a description of milestones that are related to age. In contrast to this tradition, this study seeks to account for a change and rely on the theory of Nonlinear Dynamic Systems. Therefore, using the microgenetic method, the children's ability to use the wishes of others to predict emotions
\end{abstract}


and behaviors was assessed during several sessions. Wellman and Wooley (1990) classic tasks were adapted and presented to children in 5 sessions, where there were 3 variations of the tasks. The data were analyzed with the minimum and maximum technique, finding a set of trajectories with many fluctuations, which are far from showing high and stable performances as stated in previous studies. In development research, cumulative and homogeneous views make it difficult to observe change, and its amplitude, therefore it is necessary to emphasize variability.

Keywords

desires; theory of mind; development; variability; preschool children.

Los bebés nacen en entornos sociales ricos en los que una de sus tareas más importantes es aprender a interactuar eficazmente con otras personas y apoyarse en ellas para conocer el mundo (Conte et al., 2019; Foster-Hanson \& Rhodes 2020; Tauzin \& Gergely, 2018). Para ello, es preciso que el niño comprenda que él mismo y las otras personas poseen creencias, deseos e intenciones, y que estos estados mentales sirven para explicar parte del comportamiento de los demás (Ebert, 2019; Goffin et al., 2020; Keller, 2018; Sodian \& Kristen, 2015). A esta capacidad para comprender y atribuir estados mentales a otros, y a sí mismo, se le conoce como teoría de la mente (Wellman, 2015).

\section{El caso de la teoría de la mente}

El estudio de la teoría de la mente (TdM a partir de ahora) en la primera infancia, ha alimentado la curiosidad de muchos investigadores del desarrollo cognitivo. Algunos de ellos concuerdan en que los niños a la edad de tres años saben que diferentes personas pueden desear y sentir cosas distintas, y luego, a los 4 o 5 años de edad, entienden que sus creencias pueden diferir de las de la gente (Gopnik, 2010; Wellman, 2015).

Es llamativo que la mayoría de las investigaciones en TdM infantil han dado especial preponderancia al estudio de las creencias, específicamente bajo el paradigma de la falsa creencia. No obstante, no ha ocurrido lo mismo con los deseos, que son junto con las creencias la base de la TdM (Harrigan et al., 2018; Wellman, 2015).

Los deseos son estados mentales que pueden agruparse bajo el título de 'pro' actitudes, por lo cual se consideran constructos intencionales en el sentido de tratar sobre algún objeto. Tener un deseo es básicamente una cuestión de tener disposiciones para actuar (Schroeder, 2004). Cumplen con un papel crucial en la interacción social: primero, porque muchos de los comportamientos de los seres humanos están motivados por deseos y poder comprenderlos implica a su vez comprender parte de la mente de las personas; y segundo, porque son la primera vía que tienen los bebés para explicar el comportamiento de otras personas (Asbjørn et al., 2015; Wellman; 2015). Este acercamiento a la $\mathrm{TdM}$ en relación con los deseos probablemente se vio motivado por la temprana aparición de estos últimos en los primeros meses de vida del niño, lo que se asocia con la satisfacción de necesidades fisiológicas básicas como el hambre, o el abrigo (Astington, 1993); y además, por el uso de términos mentalistas que tienen que ver con deseos desde los primeros años de vida (Ruffman et al., 2018).

De acuerdo con varios estudios, un primer momento importante en el desarrollo de la $\mathrm{TdM}$ con relación a los deseos es la capacidad de los niños pequeños (de 18 meses incluso) para comprender que otras personas pueden tener deseos distintos e incluso contradictorios a los propios (Repacholi \& Gopnik, 1997; Yott \& Poulin-Dubois, 2016). Luego, hacia los dos años y medio, los niños espontáneamente comienzan a hablar de los deseos propios y ajenos (Bretherton \& Beeghly, 1982). Parte de esa bibliografía sugiere que los infantes en sus primeras interacciones verbales utilizan verbos referidos a las emociones y alos deseos (querer, desear) antes de usar los que tienen que ver con las creencias (creer, pensar). A saber, investigaciones recientes incluso mostraron que cuando padres y cuidadores se involucran con los niños en actividades y conversaciones donde es frecuente el uso de estos términos mentalistas, los bebés pueden mostrar mejores desempeños 
en tareas que evalúan TdM en comparación con otros niños que no han estado expuestos a este tipo de interacciones (Ruffman et al., 2018)

Hacia los tres años se plantea la existencia de un modo particular que tendrían los niños para comprender el comportamiento de los otros a través de los deseos: la psicología del deseo simple (Wellman \& Wolley, 1990; Wellman, 2015). Esta propuesta sugiere que los deseos pueden clasificarse en impulsos (drives), que generalmente se refieren a las necesidades fisiológicas internas del organismo, y también en deseos simples, que aluden a un objeto o evento deseado que está presente.

Los niños de estas edades serían capaces de predecir ciertas acciones basándose en el deseo de una persona (Vaish et al., 2018). Por ejemplo, en el estudio pionero de Wellman y Woolley (1990), los niños pudieron predecir que cuando alguien alcanzaba sus deseos cesaría las acciones que lo condujeron a satisfacerlo, y también, que alguien que no satisfizo sus deseos persistirá en las acciones necesarias para alcanzarlo. Además, los niños también lograron predecir las reacciones emocionales apropiadas derivadas del deseo, esencialmente para predecir que conseguir lo que se desea produce felicidad, placer o satisfacción, y que no conseguirlo supone tristeza.

Un cuarto momento significativo que se puede identificar respecto al desarrollo de la TdM en relación con los deseos se refiere a la aparición de las creencias en el razonamiento de los niños para abordar las interacciones sociales. Algunos estudios apoyan la idea de que los niños primero hacen uso de los deseos como estados mentales antes de hacerlo exitosamente con las creencias (Harrigan et al., 2018; Steglich-Petersen \& Michael, 2015; Yott \& Poulin-Dubois, 2016; Wellman \& Wooley, 1990). Básicamente se plantea que los niños pasan por un periodo de transición que va de un razonamiento basado únicamente en los deseos, a uno en donde se incorporan las creencias. En los primeros años de vida los deseos siguen siendo los principales constructos que permiten explicar el comportamiento de los otros, y hacia el final del segundo año, comienzan a emerger las creencias como constructos auxiliares que apoyan este propósito (Steglich-Petersen \& Michael, 2015; Wellman, 2015).

Aunque usan distintas tareas, la mayoría de estudios sugieren que los niños con edades cercanas a los tres años pueden comprender los deseos simples. Además, todos ellos tienen en común la evaluación de esta capacidad por medio de diseños transversales, es decir, han basado sus conclusiones en una sola medición de la variable (Harrigan et al., 2018; Roncancio \& Puche, 2012).

Como se puede observar, a pesar de no tener tanta densidad de estudios como sucede con las creencias, los psicólogos han logrado documentar y describir hitos muy importantes en el desarrollo de los deseos en la primera infancia; sin embargo, se sabe relativamente poco respecto a qué sucede entre un hito y otro (Astington \& Edward, 2010; Roncancio \& Puche, 2012; Steglich-Petersen \& Michael, 2015). Muchas investigaciones han podido mostrar qué ocurre en momentos muy específicos del desarrollo por medio de estudios transversales. De la revisión de antecedentes, entre otras cosas, se puede concluir que se han provisto una suerte de instantáneas que muestran un antes y un después en el desarrollo; no obstante, estos estudios han fallado al describir directamente el proceso de cambio evolutivo (Vaish et al., 2018). En esencia, se han centrado en mostrar qué es lo que cambia, haciendo hincapié en fases relativamente estáticas en las cuales se analizaba la ejecución en los niños y los procesos psicológicos de base en una edad dada, para luego comparar estos hallazgos entre edades. Sólo a medida que se intentaba dar respuesta al cómo, se empezaron a incluir nuevos modelos de investigación sustentados en el microdesarrollo (Castro et al., 2012).

El enfoque transversal resulta útil cuando se toman estas instantáneas y se examinan las diferencias entre los individuos a la luz de ciertas competencias, o también, cuando se comparan las instantáneas de participantes de diferentes grupos experimentales. No obstante, no se profundiza en cómo ocurre el cambio, o qué tipo de mecanismos lo sostienen, incluyendo preguntas sobre su curso, grado y amplitud; además de sus fuentes y sus componentes (Flynn 
et al., 2006). Lo cierto es que pocos estudios han implementado métodos que permitan conocer y evaluar el desarrollo de la comprensión de deseos trascendiendo la visión estructuralista clásica; de hecho, casi todos los han abordado a partir de modelos de desarrollo con elementos estáticos, que terminan encapsulados en el estudio de estadios y estructuras definidas, y no generan nuevos conocimientos que permitan girar hacia un enfoque funcionalista de la mente infantil (Puche-Navarro, 2015; Roncancio \& Puche, 2012). Por ello, es preciso integrar en los modelos de desarrollo al cambio y la variabilidad, pues son elementos cruciales para entender el desarrollo más allá de los hitos, y en esta medida, darle relevancia al cambio intraindividual sin tener que basarse siempre en comparaciones con grupos normativos determinados por la edad.

\section{La variabilidad y el cambio son desarrollo}

Ante esta situación, una forma de abordar las preguntas sobre los procesos de cambio en el desarrollo es con los diseños microgenéticos. Estos tienen como pretensión examinar el cambio a medida que se produce, intentando identificar y explicar sus mecanismos subyacentes. Estos diseños proporcionan descripciones especialmente "gruesas" de cambios a través de evaluaciones longitudinales extendidas y estrechamente espaciadas. Se defienden como imperativo para entender cómo, en ciertas condiciones, los cambios en el micro nivel mantienen la estabilidad relativa de un sistema, mientras que en otras condiciones esos mismos cambios dan lugar al desarrollo (Puche-Navarro, 2015).

En esta línea, hay una propuesta metodológica y teórica muy pertinente para la investigación en el desarrollo apelando a la microgénesis: la perspectiva de los sistemas dinámicos. Este es un enfoque interdisciplinario usado en ciencias como la física o la biología, y que ha tomado fuerza en la psicología del desarrollo, pues proporciona un modelo para el estudio de los procesos de cambio. El enfoque hace hincapié en cómo los cambios en el micro-nivel de las relaciones entre los constituyentes de un sistema dan lugar a nuevos patrones de comportamiento a nivel macro. En consecuencia, nuevos patrones de actividad surgen de la relación mutua entre los constituyentes procedentes del individuo y del medio ambiente, y no de un plan maduracional del individuo. El desarrollo, desde esta perspectiva, se aborda y se interpreta como un sistema inestable en el cual los cambios son resultado de procesos de reorganización, más que de acumulaciones (Van Geert, 1994; Van Geert $\&$ Van Dijk, 2002).

Una de las estrategias implementadas en el estudio del desarrollo desde los sistemas dinámicos es el cálculo de trayectorias. Estas se refieren a los movimientos que acontecen en un sistema que evoluciona en una dimensión espacio-temporal, y en esa medida, dejan seguir las transformaciones y los cambios que operan en ese sistema a lo largo del tiempo (Puche-Navarro, 2015). Puche-Navarro (2015), citando a Yan y Fischer (2002) lo plantea así: "La trayectoria representa las huellas cognitivas del recorrido que sigue el sujeto momento a momento, cuando realiza una tarea cognitiva en la solución de un problema" p. 5.

Por ello, este estudio utilizó el cálculo de trayectorias para analizar cómo se daban los cambios en la comprensión de los deseos en niños preescolares entre 2 y 3 años de edad. Para ello se hizo una evaluación del rendimiento de los participantes en tareas que evaluaron uso de deseos para predicción emocional y del comportamiento, inspiradas en las que usaron Wellman y Woolley (1990).

\section{Método}

\section{Participantes}

La muestra estuvo conformada por 14 niños (5 varones) pertenecientes a instituciones públicas y privadas del oriente antioqueño. Al iniciar la evaluación tenían edades entre los 2 años y seis meses y 3 años y 0 meses. El muestreo fue intencional, determinado por la voluntad de padres de familia y participantes. No se 
incluyeron niños que tuvieran algún trastorno del desarrollo o patología que interfiera con los objetivos de la investigación. Para el desarrollo de la investigación se tuvo en cuenta el capítulo II la Resolución № 008430 de 1993 del Ministerio De Salud Nacional a partir del cual se clasifica el presente estudio en la categoría de "investigación sin riesgo". Todos los participantes contaron con la firma del consentimiento informado de sus padres, además ninguno de los experimentos puso en riesgo la integridad física y psicológica de los niños.

\section{Diseño y Materiales}

Se usó un diseño de medidas repetidas con enfoque microgenético, que implicó la realización de varias mediciones sobre las variables en periodos cortos de tiempo (Flynn et al., 2006). Para ello se utilizaron dos tareas que permitieron evaluar la comprensión de los deseos simples. Los niños participaron en un total de 5 sesiones, con evaluaciones que ocurrieron una vez por semana durante un periodo de 5 semanas. Los participantes fueron evaluados en una habitación privada de su guardería. Seis entrevistadoras fueron entrenadas para llevar a cabo las evaluaciones. Durante cada sesión los niños fueron evaluados con dos tareas, y cada una de ellas tuvo tres ensayos, para un total de 15 mediciones por tarea para cada niño, durante las cuales se contrabalanceó el orden de aplicación a través de las sesiones.

Las tareas empleadas evalúan comprensión de deseos, y estuvieron inspiradas en las diseñadas por Wellman y Woolley (1990). Una de ellas indaga por la capacidad de los niños para usar los deseos para predecir emociones, y la otra por el uso de los deseos para predecir comportamientos. Ambas tareas presentan tres situaciones: la situación de objeto deseado, donde un personaje recibe el regalo que desea (predicción emocional) o encuentra el objeto que deseaba (predicción del comportamiento). La situación sin objeto, en la cual el personaje desea un regalo, y no recibe nada (predicción emocional) o desea encontrar un objeto para un propósito y en el lugar que lo busca no lo encuentra (predicción del comportamiento). Por último, la situación sustituto, en la que el personaje recibe un regalo que no es acorde a sus preferencias o encuentra un objeto que no se corresponde con el que desea llevar a un lugar determinado.

Tanto en la tarea de predicción emocional como en la de predicción comportamental, sólo las situaciones que implican recibir o encontrar el objeto deseado se corresponden con la satisfacción del deseo y la alegría, mientras que las situaciones sustituto y sin objeto implican la insatisfacción del deseo y en consecuencia, la tristeza. Por esta razón, en todos los ensayos de las dos tareas mencionadas hubo una situación donde el deseo fue satisfecho y otra donde fue insatisfecho.

Para la tarea de predicción emocional se utilizaron juguetes que sirvieron como objetos deseados: unas crayolas, una bicicleta, una pelota y unas llaves. Además de una tarjeta que mostraba un personaje (niño o niña según el género del participante), que se encontraba de espaldas para evitar que la expresión facial del dibujo indujera algún tipo de respuesta en el niño. También se utilizaron 2 tarjetas adicionales que representaron la expresión emocional de la alegría y la tristeza de un niño y una niña (se utilizaron los rostros que se corresponden con el género del participante).

Por otro lado, en la tarea de predicción del comportamiento se usaron 3 cajas de $6 \times 6 \times 6 \mathrm{~cm}$ con tapas que tenían la imagen de tres lugares correspondientes a la sala y el patio de una casa, y a una escuela. También se utilizó la tarjeta con el personaje de espaldas, utilizado en la tarea de predicción emocional. Además, tarjetas con objetos cotidianos para los niños, y similares entre sí por características generales, como un perro, un gato y un conejo (mascotas). Dichas tarjetas tenían dimensiones que permitieron introducirse en las cajas, y fueron fácilmente identificables por los niños. 


\section{Procedimiento}

\section{Tarea predicción emocional}

Inicialmente se le pidió al participante que identificara la expresión emocional de dos tarjetas: una con la imagen de un rostro feliz y la otra con un rostro triste; para ello se hizo el siguiente requerimiento: "aquí hay un niño(a) que está triste y otro que está feliz. ¿Cuál de ellos está triste y cuál está feliz?". El niño podría responder verbalmente o señalando el rostro del personaje, y antes de comenzar con la tarea se garantizó que identificara correctamente ambas expresiones emocionales. Posteriormente se le dijo al niño que había un personaje que deseaba un objeto, y se hizo la pregunta control "iQué es lo que desea X?". Posterior a la pregunta control y de estar seguros que el niño había entendido la consigna, se continuaba con la tarea de comprensión de deseos. Por último, siempre se le pidió al niño que dijera o señalara en las tarjetas cuál era la reacción emocional del personaje luego de recibir el objeto.

En la situación objeto deseado se le dijo al niño: "X quiere que su papá le regale este juguete" (se nombra al objeto); .él desea mucho que le den esto, y su papá se lo regala", (se usaron la pelota, la bicicleta y las crayolas). Inmediatamente hecha la pregunta control: "iqué es lo que desea X?" y que se garantizara que el niño respondiera de forma correcta, se le preguntaba al niño: "iCuándo X reciba este juguete (nombrar el objeto correspondiente) se va a sentir feliz o triste?".

Para la situación sustituto se realizó exactamente lo mismo que en la versión objeto deseado, solo que en este caso no se dio el regalo deseado al personaje, sino otro objeto. Por último, en la situación sin objeto se realizó exactamente lo mismo que en la situación objeto deseado, solo qué en este caso, el personaje no recibió ningún regalo.

\section{Tarea predicción del comportamiento}

En esta tarea había un personaje que quería encontrar un objeto (un perro, por ejemplo) para un propósito específico; en este caso llevarlo a la escuela. El objeto que deseaba podía estar en una de dos ubicaciones: el patio o la sala de su casa. El personaje, que era manipulado por la experimentadora, siempre buscaba el objeto deseado en la ubicación 1 (la sala, por ejemplo) y podía encontrarlo o no. Posterior a ello, al participante se le plantearon dos acciones que podía realizar el personaje, que se derivan de haber encontrado o no encontrado el objeto deseado: seguir buscando el objeto o ir con el objeto a otro sitio. Los niños tuvieron que elegir cuál era la acción correcta en caso que el personaje encontrara o no su objeto deseado, es decir: seguir buscando, o ir al otro lugar (la escuela). Es importante añadir que la inclusión de la situación del hallazgo de un objeto sustituto en los procedimientos fue vital para evaluar si los niños comprendían la especificidad del objeto que deseaba el personaje.

La tarea comenzaba mostrándole al participante los lugares que hacían parte de la tarea: dos cajas que tenían en su cara superior las imágenes de la sala y el patio de la casa, los cuales fueron ubicadas a una distancia de $5 \mathrm{~cm}$ una de la otra, y en el extremo opuesto la caja que representaba la escuela. El experimento tuvo tres situaciones y en todas ellas se hicieron dos preguntas de control para garantizar que el niño tuviera toda la información que requería para desenvolverse bien en la tarea. Las preguntas fueron: “¿Cuál animal quiere encontrar X?" y "¿Para qué quiere encontrar al animal (perro/ gato/conejo)?"

$\mathrm{Al}$ igual que la tarea para predicción emocional, esta contó con tres situaciones. En la situación encuentra objeto deseado, a los niños se les dijo: "este es $\mathrm{X}$, él quiere encontrar a su mascota (perro/gato/conejo) para llevarlo a la escuela, la mascota (se nombra al animal) puede estar escondido en dos lugares, el patio o la sala de la casa" (se señala y se menciona). Estos lugares están representados en cajas tapadas, 
que contienen las tarjetas con imágenes de los animales. En ese momento se hacían las preguntas de control: "iqué es lo que quiere encontrar X?", y "ipara qué quiere encontrarlo?" Luego se mostraba al personaje dirigiéndose a uno de los lugares, donde abría una de las cajas en la cual podía estar la mascota. Después que el personaje revisaba en el primer lugar, los niños debían hacer sus predicciones verbalmente, o indicando su elección señalando. Para poder juzgar el desempeño de los niños, se les preguntó: "¿Qué va a hacer X ahora?”, “¿Buscará en el otro lugar a su mascota o va a ir a la escuela?".

Por su parte, la situación sin objeto fue exactamente igual a la anterior, solo que al buscar en la ubicación 1, el personaje no encontraba ningún objeto. Es importante mencionar que cuando esta precede a la situación encuentra objeto deseado, se debe asegurar de haber extraído de la caja el objeto que representa la ubicación 1 , pues al ejecutar esta versión, no debe haber nada allí. Por último, la situación encuentra sustituto es similar a la de encuentra objeto deseado, solo que al niño se le mencionaba que el personaje deseaba encontrar un animal distinto al que hallaba dentro de la caja correspondiente a la ubicación 1. Es decir, si el personaje deseaba encontrar por ejemplo, al perro, al buscar en la ubicación 1 encontraba al gato o al conejo.

A continuación, se presenta el análisis que especifica los desempeños que se les asignaron a los niños según sus respuestas en las tareas, y los posibles funcionamientos cognitivos de base para cada nivel de desempeño.

\section{Tabla 1}

Sistema de calificación y funcionamiento en tarea de uso de deseos para predicción emocional y del comportamiento

\begin{tabular}{|c|c|c|}
\hline Tipo de desempeño & $\begin{array}{l}\text { Funcionamiento tarea } \\
\text { predicción emocional }\end{array}$ & $\begin{array}{c}\text { Funcionamiento tarea predicción } \\
\text { comportamental }\end{array}$ \\
\hline $\begin{array}{l}\text { Desempeños bajos: } \\
\text { fluctuación entre los } \\
\text { puntajes } 1,2 \text { y } 3 \text { a lo } \\
\text { largo de las mediciones }\end{array}$ & $\begin{array}{l}\text { E1 niño parece elegir de forma } \\
\text { aleatoria u orientado por su propio } \\
\text { deseo. No se evidencia que logre } \\
\text { discriminar entre la satisfacción o } \\
\text { insatisfacción de los deseos del } \\
\text { personaje y la emoción asociada a } \\
\text { cada una de estas posibilidades. }\end{array}$ & $\begin{array}{l}\text { El niño parece elegir de forma } \\
\text { aleatoria u orientado por su propio } \\
\text { deseo. No se evidencia que logre } \\
\text { discriminar entre la satisfacción o } \\
\text { insatisfacción de los deseos del } \\
\text { personaje y la emoción asociada a } \\
\text { cada una de estas posibilidades. }\end{array}$ \\
\hline $\begin{array}{l}\text { Desempeños medios: } \\
\text { fluctuación entre los } \\
\text { puntajes } 3,4 \text { y } 5 \text { a lo } \\
\text { largo de las mediciones }\end{array}$ & $\begin{array}{l}\text { Se presentan indicios de uso } \\
\text { adecuado del deseo para predecir } \\
\text { las emociones, aunque sigue } \\
\text { siendo ambiguo e intermitente y } \\
\text { no logran concretarse, pues el niño } \\
\text { aún no elige la emoción correcta } \\
\text { en ambas situaciones. La elección } \\
\text { de la alegría implica niveles menos } \\
\text { complejos que la elección de } \\
\text { tristeza, pues es más probable que } \\
\text { el niño seleccione esta alternativa, } \\
\text { asumiendo que el hecho de recibir } \\
\text { cualquier regalo (así no sea el } \\
\text { deseado por el personaje) } \\
\text { generaría alegría. }\end{array}$ & $\begin{array}{l}\text { Se presentan indicios de uso } \\
\text { adecuado del deseo para predecir } \\
\text { comportamientos, aunque sigue } \\
\text { siendo ambiguo e intermitente y no } \\
\text { logran concretarse, pues el niño aún } \\
\text { no elige el comportamiento correcto } \\
\text { en ambas situaciones. Predecir el } \\
\text { comportamiento al encontrar un } \\
\text { sustituto requiere mayor nivel de } \\
\text { análisis por parte del niño, pues debe } \\
\text { recordar que el deseo del personaje } \\
\text { solo se satisface al encontrar ese } \\
\text { objeto específico. Encontrar un } \\
\text { objeto deseado o de no encontrar } \\
\text { nada, implicaría menor complejidad. }\end{array}$ \\
\hline $\begin{array}{l}\text { Desempeños altos: } \\
\text { fluctuación entre los } \\
\text { puntajes } 5,6 \text { y } 7 \text { a lo } \\
\text { largo de las mediciones }\end{array}$ & $\begin{array}{l}\text { El niño da muestras de estar } \\
\text { usando adecuadamente los deseos } \\
\text { para predecir alegría cuando estos } \\
\text { son satisfechos, y predecir tristeza } \\
\text { cuando son insatisfechos. Se } \\
\text { evidencian mecanismos } \\
\text { cognitivos complejos para la tarea } \\
\text { como la toma de perspectiva y la } \\
\text { inferencia. }\end{array}$ & $\begin{array}{l}\text { El niño da muestras de usar } \\
\text { adecuadamente los deseos del } \\
\text { personaje para predecir el } \\
\text { comportamiento. Predice cese de } \\
\text { búsqueda del objeto cuando el deseo } \\
\text { es satisfecho, y continuación de la } \\
\text { búsqueda del objeto cuando el deseo } \\
\text { es insatisfecho. }\end{array}$ \\
\hline
\end{tabular}

\section{Resultados}

En total se evaluaron 14 participantes, 5 niños (35\%) y 9 niñas (65\%) con edades entre los 2 años y seis meses y los tres años al momento de iniciar las evaluaciones. De cada uno de los participantes se obtuvieron 15 medidas por tarea, para un total de 30 medidas. Para cada una de las tareas se graficó una trayectoria por medio de la técnica de mínimos y máximos. Esta técnica permite estudiar la tendencia del desarrollo, y a su vez la variabilidad en torno a una tendencia general por medio de un gráfico de movimientos. Esta técnica muestra los datos utilizando el ancho de banda de los puntajes observados. En lugar de mostrar los puntos de medición como puntos simples, el gráfico de movimiento mínimo-máximo presenta un rango de puntuación para cada ocasión de medición. (van Geert \& van Dijk, 2002). 
En la Figura 1 se presenta un ejemplo de trayectoria obtenida a partir de la técnica, mostrando el desempeño de uno de los participantes en la tarea de predicción del comportamiento, donde se evidencia la presencia de movimientos irregulares con muchos altibajos que no llegan a estabilizarse a lo largo de las 15 medidas obtenidas.

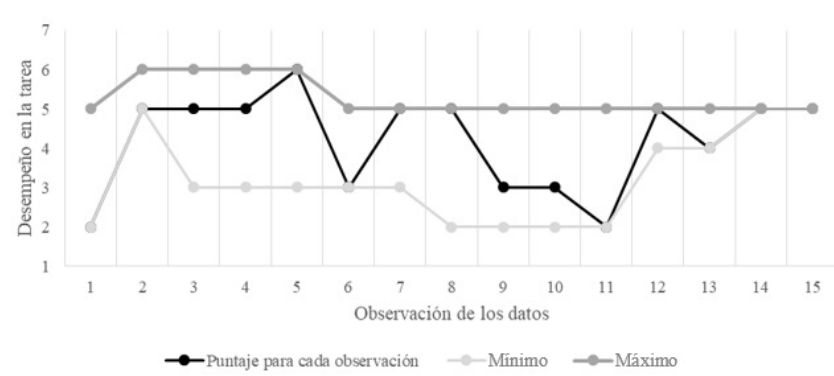

Figura 1

Ejemplo de trayectoria

\section{Desempeños en la tarea de predicción emocional}

Luego de hacer un análisis de las gráficas que mostraban las trayectorias de cada niño en las dos tareas, fue posible agruparlas en cuatro categorías que responden a una combinación de elementos comunes de patrones que involucran desempeños bajos, medios altos o irregulares (fluctuaciones constantes que nunca llegan a estabilizarse), y también patrones de alta variabilidad, poca variabilidad y estabilidad. En la Figura 2 se muestran los desempeños por tipo de trayectoria de los 14 niños en la tarea de predicción emocional. E1 corresponde a la categoría desempeños altos con patrón de poca variabilidad, E2 a desempeños bajos con patrón de poca variabilidad, E3 a desempeños medios con patrón de poca variabilidad y E4 a desempeños irregulares con patrón de alta variabilidad. En este caso, observamos que no hubo una trayectoria que se destacara por tener mayor frecuencia que el resto. No hubo una tendencia marcada que sobresaliera, lo que indica que existieron múltiples posibilidades de desempeño. No obstante, las trayectorias E1,
E2 y E3 tienen en común la presencia de patrones de poca variabilidad. Tal parece que los niños mostraron diversas "rutas" o maneras de enfrentarse a la tarea que se reflejan en tal igualdad de posibilidades de desempeños. Esta era una tarea donde esperábamos ver resultados marcados por puntajes altos en la mayoría de casos, tal como sugiere la mayor parte de la bibliografía.

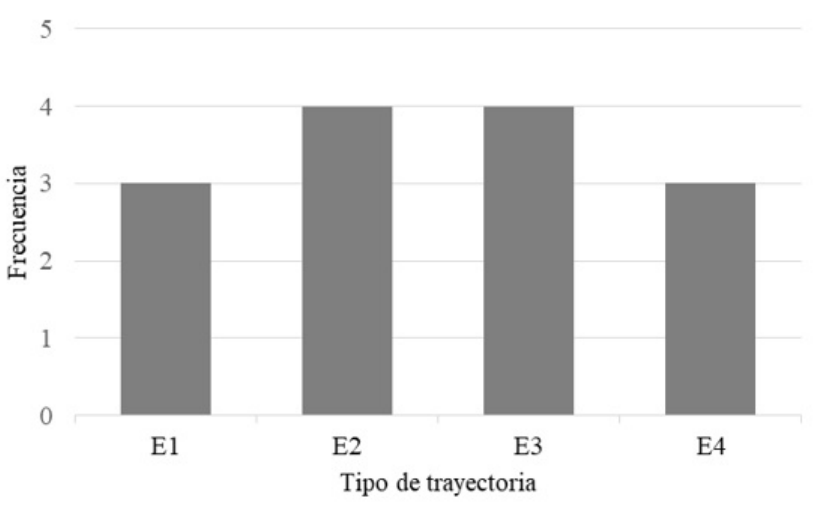

Figura 2

Trayectorias en uso de deseos para predicción emocional

Desempeños en la tarea de predicción comportamental

En la Figura 3 se consignan los resultados en la tarea de predicción del comportamiento, en donde $\mathrm{C} 1$ corresponde a desempeños altos con patrón de poca variabilidad, C2 a desempeños bajos con patrón de poca variabilidad, C3 a desempeños medios con patrón de poca variabilidad y $\mathrm{C} 4$ a desempeños irregulares con patrón de alta variabilidad. Claramente se observa la predominancia de dos tipos de trayectorias: por un lado, la trayectoria C3 que corresponde a desempeños medios con patrón de poca variabilidad, y también la trayectoria $\mathrm{C} 4$, que implica desempeños irregulares con patrón de alta variabilidad. Esta irregularidad en los desempeños dista mucho de la alta proporción de éxito que tuvieron los niños evaluados por Wellman y Woolley (1990) en la misma tarea. 


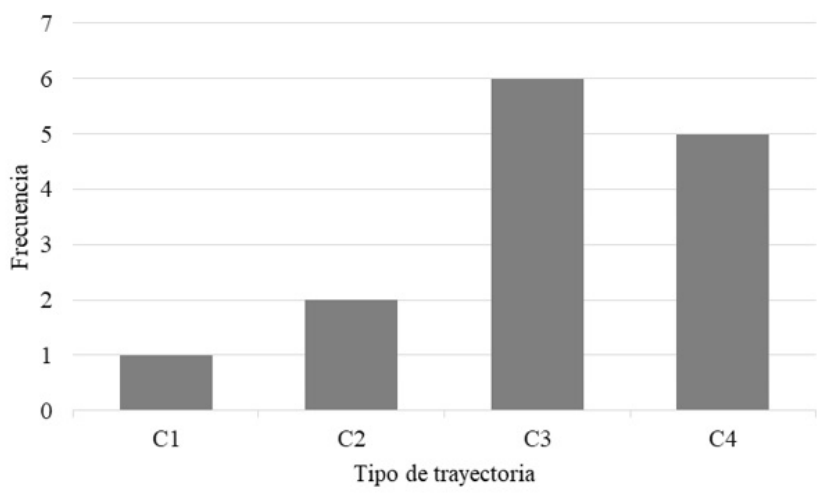

Figura 3

Trayectorias en uso de deseos para predicción del comportamiento

Desempeño global en la comprensión de deseos.

Es indiscutible que los participantes de esta investigación lejos de mostrar puntajes altos y constantes durante todas las mediciones, presentaron movimientos muy importantes que se deben considerar. Si comparamos en paralelo los tipos de trayectoria en las tareas de predicción emocional y comportamental por medio de los deseos, notaremos ciertas diferencias, en especial en el tipo de trayectorias E1 y C1 que corresponden a desempeños altos con poca variabilidad, y también en las trayectorias E2 y $\mathrm{C} 2$, que describen desempeños medios con poca variabilidad. El desempeño de los niños no fue equivalente en estas dos tareas, esto da un primer indicio de que se podrían estar evaluando dos dominios distintos del fenómeno.

Por último, la Figura 4 presenta en paralelo la proporción de los desempeños de los niños en todas las tareas, que pudimos clasificar en cuatro categorías: desempeños altos, desempeños medios, desempeños bajos y desempeños irregulares.

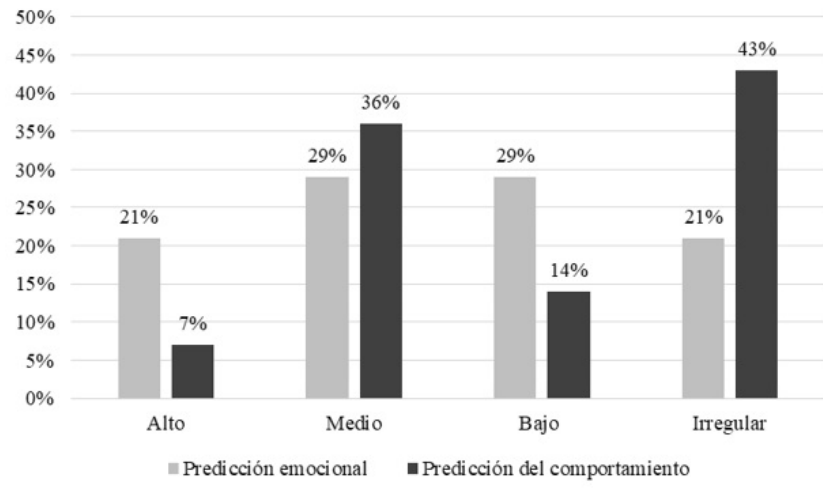

Figura 4

Proporción del tipo de desempeño en las tareas

Respecto a este análisis, lo primero que hay que mencionar es que menos de la cuarta parte de los niños tuvo desempeños altos o ideales en cualquiera de las dos tareas, de hecho, en la que tuvieron rendimientos más bajos fue en la tarea de predicción del comportamiento. En lo que respecta a las tareas de uso del deseo para predicción emocional y del comportamiento, la literatura sugiere que los niños de estas mismas edades presentan desempeños altos. Por otra parte, llama la atención la alta proporción de desempeños irregulares marcados por la alta variabilidad, especialmente en las tareas de predicción del comportamiento. Esto indicaría que los niños mostraron altibajos muy marcados respecto a sus puntajes, específicamente una tercera parte en la de predicción del comportamiento y una quinta parte en la tarea de predicción emocional.

\section{Discusión}

El objetivo de este estudio fue el de analizar cómo se dan los cambios en la comprensión de los deseos en niños preescolares. Para ello se emplearon dos situaciones experimentales diseñadas por Wellman y Woolley (1990), las cuales permitieron evaluar la capacidad de los niños para predecir las emociones y el comportamiento en la medida en que los deseos 
Juan Pablo Arroyave Hurtado, Johny Villada Zapata, Milton Eduardo Bermudez Jaimes.

de un personaje fueran satisfechos o no. El análisis de cada una de las trayectorias permitió establecer varios tipos de desempeños en cada una de las variables estudiadas, en el que fue llamativa la presencia de la variabilidad en los desempeños de la mayoría de los niños. Esta variabilidad permitiría inferir que, en lo que respecta a la comprensión de los deseos, no se puede concluir tajantemente que los niños entre los 2 y los 3 años puedan comprender deseos simples con tal fluidez como sugiere gran parte de la bibliografía sobre el tema.

Las fluctuaciones presentes a lo largo de una misma tarea dan cuenta de la variabilidad intraindividual y no de desempeños homogéneos. En la mayoría de los casos el desempeño de los niños no se correspondió con trayectorias lineales ascendentes como se esperaría ante una posible familiarización con las tareas. Lo que parece que sucedió, es que el niño estuvo explorando distintos caminos que en un momento consideró y en otro omitió, algo así como una tensión entre los mecanismos cognitivos que le permitirían comprender la tarea (Guevara Guerrero \& Puche-Navarro, 2009). Es importante recordar que el modo de calificación no fue dicotómico, restringiendo la respuesta del niño a correcto o incorrecto, sino que por medio de análisis de tarea se establecieron unos puntajes que se otorgaron al participante según su desempeño, donde se asume que la respuesta que da cada niño ante las tareas tiene como base procesos cognitivos de diversa complejidad, esto es, los puntajes bajos presumiblemente son el reflejo de una búsqueda aleatoria u orientación por el propio deseo, y los puntajes más altos reflejan el uso de mecanismos inferenciales sofisticados que permiten resolver la tarea de manera exitosa (Roncancio \& Puche-Navarro, 2012)

Ahora bien, desde la teoría de Sistemas Dinámicos aplicados al desarrollo cognitivo también encontramos razones para analizar nuestros resultados, enmarcados mayoritariamente por la presencia de mucha variabilidad en los desempeños de los niños en las dos tareas. Por ejemplo, observamos que los tipos de trayectoria E3 y C3 (desempeños medios con patrón de poca variabilidad) y E4 y C4 (desempeño irregular con patrón de alta variabilidad) fueron los más comunes en toda la evaluación. Por un lado, se podría pensar que las trayectorias que tuvieron poca variabilidad, en las que los desempeños fueron muy similares en todas las mediciones, estén dando cuenta de algunas preferencias conductuales, que les confiere cualidades propias de un estadio evolutivo, por ejemplo, aquellos niños que solo mostraron desempeños medios o bajos a lo largo de todas las mediciones. No obstante, la estabilidad se asume en este caso como función del organismo en contexto y estos estados son patrones conductuales preferentes, y altamente probables para posteriores mediciones, pero no obligatorios. Por esta razón insistimos en que una sola medida no puede ser predictor ideal del comportamiento futuro, pues ni siquiera quince, para nuestro caso particular, logran ese propósito.

Por otra parte, en el caso opuesto, en donde la constante fue la alta variabilidad en los desempeños, pareciera que esa preferencia conductual mostrara cierta perturbación, es decir, las necesidades adaptativas del sistema le estarían conduciendo a un nuevo estado y de esa manera se podría explicar el cambio. Tuvimos casos en los que niños en la misma tarea presentaron puntajes muy bajos, probablemente porque estaban eligiendo al azar o usando una estrategia de ensayo y error, pero también encontramos puntajes altos que nos darían cuenta de mecanismos inferenciales más complejos. Desde esta perspectiva, la dimensión temporal podría explicar este funcionamiento, por lo que en distintos momentos las mismas condiciones pueden generar resultados diferentes. De esta forma, en el desarrollo natural, un estado precede a otro y lo condiciona, dibujando el paisaje epigenético del desarrollo individual (van Geert, 1994 citado en Abellán et al., 2015).

Encontramos que cerca del $21 \%$ de los participantes tuvieron desempeños altos a lo largo de todas las mediciones en la tarea de predicción emocional, hecho que contrario los hallazgos de buena parte de la literatura científica que ha abordado el tema, pues se había afirmado que los niños preescolares eran 
capaces de predecir correctamente la emoción de alguien teniendo en cuenta la satisfacción o no satisfacción de su deseo (Hadwin \& Perner, 1991; Wellman \& Wooley, 1990). Nuestros hallazgos en esta variable en particular nos llevan a pensar que los niños no parecen razonar fluidamente con el concepto de deseo en lo referente a la predicción emocional ya que, aunque algunos de ellos obtuvieron desempeños altos en alguna de las mediciones, la constante fue encontrar desempeños medios y bajos (29\% en cada caso), y desempeños irregulares (21\%).

En las tareas de predicción emocional, en promedio los niños acertaron el 70\% de los casos en las situaciones que exigían predecir la alegría del personaje cuando su deseo era satisfecho, mientras que en la predicción de la tristeza producto de la insatisfacción del deseo, el promedio de aciertos fue del $24 \%$. Estos datos distan de los hallazgos de Wellman y Wooley (1990) quienes reportaron una tasa de éxito superior al $85 \%$ en ambas situaciones. Utilizar una medida de resumen, en este caso el promedio en la proporción de aciertos para predecir alegría o tristeza, brinda información importante pero insuficiente para comprender y explicar qué sucede en el desarrollo cognitivo cuando hablamos del uso de un estado mental definido por el deseo para predecir las emociones. Decir que el $70 \%$ de la muestra acertó es quedarse a medio camino e ignorar valiosa información que puede inferirse de los datos que recolectamos, pues la media pondera todos los valores, pero omite los detalles más minuciosos del desempeño y desarrollo de cada niño al dejar de lado elementos cruciales como la variabilidad y el cambio. Si hubiésemos optado por un plan de análisis y metodología tradicional, no podríamos decir mucho sobre el desempeño de los participantes a lo largo del tiempo y, en consecuencia, hubiera sido muy complicado evaluar el desarrollo de la comprensión de deseos con sus múltiples manifestaciones en cada niño.

Una posible respuesta de por qué los participantes presentaron dificultades al momento de usar los deseos para predecir la emoción cuando éstos no se cumplían, podría ser lo que algunos autores han llamado sesgo de alegría (Rieffe et al., 2001). Cuando la emoción acorde con los deseos es positiva, los niños tendrían un mejor desempeño a cuando la emoción es negativa, lo que indica una tendencia a predecir emociones de alegría con mayor frecuencia (Rieffe et al., 2001, p.271 en Riviere et al., 2014). Algunas investigaciones evaluaron a niños de cuatro y cinco años en tareas que implicaban predecir emociones a partir de los deseos de personajes que iban en contravía de los del niño (Riviere et al., 2014) o también predicción de emociones cuando sus deseos coincidían o no con los estereotipos sociales de género (Rieffe et al., 2001), y en ambas fue innegable la preferencia por elegir una emoción de valencia positiva en lugar de una negativa (en este caso, la alegría por la tristeza). Esta tendencia a hacer predicciones de emoción positiva, podría hacerse más evidente cuando están implicados los propios deseos y emociones de los participantes, es decir, cuando el niño se orienta por su propio deseo. En nuestro caso, la tarea exigía al niño predecir la emoción de un personaje del cual se había hecho explícito su deseo por un juguete. Realmente es complejo demostrar que el niño respondió en función del deseo del personaje, pues cabe la posibilidad de que él también deseara el objeto que se estaba utilizando en ese momento; sin embargo, las preguntas de control brindan cierta garantía de que el participante sabe qué es lo que desea el personaje.

Por otra parte, al centrarnos en la tarea de predicción del comportamiento, que según Wellman (2015) también haría parte de la psicología del deseo simple junto con la predicción emocional, encontramos que el $43 \%$ de los niños tuvo desempeños muy irregulares con alta variabilidad, y el $36 \%$ mostró desempeños medios con patrones de poca y alta variabilidad, lo cual claramente refleja una diferencia con los supuestos teóricos clásicos. Incluso, si se hace el mismo ejercicio de comparación de la tasa de éxito en las respuestas, se puede notar que los participantes tuvieron promedios de acierto mucho más bajos en relación con los niños que estuvieron en el estudio de Wellman y Wooley (1990) que 
acertaron en el $85 \%$ de los casos usando la misma tarea.

Esta tarea pudo haber sido más compleja que la de predicción emocional pues involucró muchos más elementos en escena. El niño debía tener en cuenta que había tres lugares igualmente importantes en la situación, dos en donde podía estar el animal que el personaje deseaba encontrar, y otro a donde lo llevaría en caso de hallarlo. A pesar de que los niños respondían acertadamente a las preguntas de control "¿Cuál animal quiere encontrar X?" y "ipara qué quiere encontrar al animal?", la demanda cognitiva en este caso puede haber sido superior a la de la tarea de predicción emocional, donde los niños solo debían tener en cuenta el deseo del personaje por un objeto, y luego predecir su reacción emocional. Aquí el niño no solo debía tener en cuenta el deseo del personaje, sino también conservar en su mente la acción subsecuente, es decir, tenía que recordar para qué quería el personaje encontrar, por ejemplo, al conejo.

Esto es importante porque los deseos que se utilizan en la tarea de predicción del comportamiento pueden ser considerados como deseos instrumentales, pues sirven como un medio para algún otro fin, distinto a los deseos intrínsecos, que son estados de cosas que se buscan por sí mismas (Schroeder, 2004). En la tarea de predicción emocional al niño se le decía que el personaje deseaba una pelota, o una patineta, pero no se mencionaba ningún propósito para tener ese deseo: el personaje simplemente quería que le regalaran ese juguete (deseo intrínseco), mientras que en la tarea de predicción del comportamiento era fundamental que el niño tuviera en cuenta el propósito de encontrar al animal deseado, que era llevarlo a la escuela (deseo instrumental). Además, también es probable que presentaran dificultades tanto en esta tarea como en la de predicción emocional porque en ambas se requería que realizaran una toma de perspectiva de la respuesta del personaje, es decir, el niño debía inhibir su propio deseo para centrarse en lo que debería hacer el personaje de la historia, o en cuál debía ser su reacción emocional.

\section{Conclusiones}

Una de las conclusiones que deja el estudio es que cada niño lleva un proceso particular, y esto ratifica la necesidad de tener más mesura al momento de generalizar los casos, y mucho más al pretender establecer un criterio normativo determinado por la edad con el objetivo de demarcar hitos en el desarrollo de la comprensión de los deseos, y del desarrollo cognitivo en general. A partir de los datos se puede notar que muchos de los participantes podían tener desempeños altos y bajos independientemente de su edad; por ejemplo, algunos de los niños más pequeños (2 años y seis meses) tuvieron desempeños altos en algún momento de sus evaluaciones; asimismo, encontramos desempeños bajos en los niños más grandes (3 años, por ejemplo).

Luego de este recorrido, lo que se puede anotar es que el uso de los deseos para predecir las emociones y el comportamiento es un proceso más complejo de lo que sugiere gran parte de la literatura. En primer lugar, es evidente la presencia de variabilidad y la existencia de varios tipos de desempeños en los niños, por lo tanto, nuestra propuesta metodológica cobra mucho más valor, pues nuestros datos permitieron mostrar que el desarrollo implica variabilidad, y dicha variabilidad puede aportar elementos para explicar el cambio y el funcionamiento cognitivo en cualquiera de sus ámbitos específicos.

Por otra parte, no creemos que sea correcto afirmar categóricamente que en determinada edad (por lo regular a los tres años) los niños sean capaces de predecir emociones y conductas usando los deseos o que pueden inferirlos sin problema, pues lo que muestran nuestros resultados es que no hay un patrón de repuesta estable, ni tampoco exitoso en todas las mediciones. Hay que intentar trascender al esquema del desarrollo a partir de una ruta única como acceso al conocimiento, y abrir una ventana que contemple mayor variedad de rutas. El desarrollo realmente está lejos de ser lineal, en el sentido de que se mire como acumulativo o creciente, más bien aparece 
desigual, heterogéneo, y como se decía antes, marcado por la diversidad de rutas.

\section{Referencias}

Abellán, F., Calvo-Llena, M., \& Rabadán, R. (2015). Escala de desarrollo armónico. Una propuesta integradora para la evaluación del desarrollo infantil. Anales de Psicología, 31(3), 837-848. https://doi.org/10.6018/an alesps.34.1.287611

Asbjørn Steglich, P., \& Michael, J. (2015) Why Desire Reasoning is Developmentally Prior to Belief Reasoning. Mind $\mathcal{E}$ Language, 30(5), 526-549. https://doi.org/10.1111/mi la.12089

Astington J. W. (1993). The child's discovery of the mind. Harvard University Press. https://www.hup.harvard.edu/catalo g.php?isbn $=9780674116429$

Astington, J., \& Edward, M (2010) The Development of Theory of Mind in Early Childhood. Encyclopedia of infant and early childhood development. https://www.child-encyclope dia.com/sites/default/files/textes-experts/e $\mathrm{n} / 588 /$ the-development-of-theory-of-mind -in-early-childhood.pdf

Bretherton, L. \& Beeghly, M (1982). Talking about internal states: the acquisition of an explicit theory of mind. Developmental Psychology, 18, 906-921. https://doi.org/10. 1037/0012-1649.18.6.906

Castro, J., Sierra, H., \& Flórez-Romero, R. (2012). Una revisión de las relaciones entre los sistemas dinámicos y la psicología del desarrollo. Suma Psicológica, 19(2), 109-130. https://www.scielo.org.co/pdf/su mps/v19n2/v19n2a09.pdf

Conte, E., Ornaghi, V., Grazzani, I., Pepe, A., \& Cavioni, V. (2019). Emotion Knowledge, Theory of Mind, and Language in Young Children: Testing a Comprehensive Conceptual Model. Frontiers in Psychology. 10, 2144. https://doi.org/10.3389/fpsyg.201 9.02144
Ebert, S. (2019). Theory of mind, language, and reading: Developmental relations from early childhood to early adolescence. Journal of Experimental Child Psychology, 191, 1-20. https://doi.org/10.1016/j.jecp.20 19.104739

Flynn, E., Pine, P., \& Lewis, C. (2006). The microgenetic method: Time for change? The Psychologist, 19(3). https://thepsychologist.bps.org.uk/v olume-19/edition-3/microgenetic-methodtime-change

Foster-Hanson, E., \& Rhodes, M. (2020) How origin stories shape children's social reasoning. Cognitive Development, 56, 100962. https://doi.org/10.1016/j.cogdev.2 020.100962

Goffin, K., Kochanska, G., \& Eunyoon, J. (2020). Children's theory of mind as a mechanism linking parents' mindmindedness in infancy with children's conscience. Journal of Experimental Child Psychology, 193, 1-14. https://doi.org/10.10 16/j.jecp.2019.104784

Gopnik, A. (2010). El bebé filosófico. México: Editorial Planeta. https://doi.org/10.1080/1 3698036.2010.488018

Guevara, M., \& Puche-Navarro, R. (2009). iSe desarrolla y cambia la psicología del desarrollo hacia los sistemas dinámicos no lineales? Avances en Psicología Latinoamericana, 27(2), 327-342. https://revistas.urosario.edu.co/in dex.php/apl/article/view/1178/1048

Hadwin, J., \& Perner, J. (1991). Pleased and surprised: Children's cognitive theory of emotions. British Journal of Developmental Psychology, 9, 215-234. https://doi.org/10.1 111/j.2044-835X.1991.tb00872.x

Harrigan, K., Hacquard, V., \& Lidz, J. (2018) Three-Year-Olds' Understanding of Desire Reports Is Robust to Conflict. Frontiers in Psychology, 19(119), 1-13. https://doi.org/1 $0.3389 /$ fpsyg.2018.00119

Keller, H. (2018) Parenting and socioemotional development in infancy and early childhood. Developmental Review, 50, 31-41. https://doi.org/10.1016/j.dr.2018.03.001 
Juan Pablo Arroyave Hurtado, Johny Villada Zapata, Milton Eduardo Bermudez Jaimes.

Ministerio de Salud. (1993). Resolución № 008430 de 1993. Colombia: Autor.

Puche-Navarro, R., (2015) El desarrollo cognitivo se reorganiza. Bogotá. California edit. https://california-edit.com/libro/el-des arrollo-cognitivo-se-reorganiza-emergencia -cambio-metaforas-visuales-autoregulacion /

Repacholi, B. M., \& Gopnik, A. (1997). Early reasoning about desires: Evidence from 14- and 18-month-olds. Developmental Psychology, 33, 12-21 https://psycnet.apa.or g/doi/10.1037/0012-1649.33.1.12

Rieffe, C. J., Meerum Terwogt, M., Koops, W., Stegge, G. T. M., \& Oomen, A. (2001). Preschoolers appreciation of uncommon desires and subsequent emotions. British Journal of Developmental Psychology, 19(2), 259-274. https://doi.org/10.1348/02615100 1166065

Rivière, A., Arias, L., \& Sarriá, E. (2014) Emotional prediction in desire mismatching or matching situations. Infancia $y$ Aprendizaje, 30(3), 325-341. https://doi.org /10.1174/021037007781787453

Roncancio, M., \& Puche-Navarro, R. (2012). Humor gráfico y comprensión de deseos. Diversitas: Perspectivas en Psicología, 8(2), 345-360. https://doi.org/10.15332/s1794-9 998.2012.0002.10

Ruffman, T., Puri, A., Galloway, O., Su, J., \& Taumoepeau, M. (2018). Variety in parental use of "want" relates to subsequent growth in children's theory of mind. Developmental Psychology, 54(4), 677-688. h ttps://doi.org/10.1037/dev0000459

Schroeder, T. (2004). Three faces of desire. New York: Oxford University Press. En E. Zalta (ed.) The Stanford Encyclopedia of Philosophy, https://plato.stanford.edu/archi ves/sum2017/entries/desire

Sodian, B., \& Kristen, S (2015). Theory of Mind During Infancy and Early Childhood Across Cultures, Development of. International Encyclopedia of the Social $\mathcal{B}$ Behavioral Sciences, 24(2), 268-273. https://doi.org/10. 1016/B978-0-08-097086-8.23077-8
Steglich-Petersen, A., \& Michael, J. (2015). Why Desire Reasoning is Developmentally Prior to Belief Reasoning. Mind $\mathcal{E}$ Language, 30(5), 526-549. https://doi.org/10.1111/mil a.12089

Tauzin, T., \& Gergely, G. (2018). Communicative mind-reading in preverbal infants. Scientific reports, 8(1), 1-9. https://doi.org/10.1038/s4 1598-018-27804-4

Vaish, A., Hepach, R., \& Grossmann, T. (2018). Desire understanding in 2-yearold children: An eye-tracking study. Infant Behavior and Development, 52, 22-31. https ://doi.org/10.1016/j.infbeh.2018.05.002

van Geert, P. (1994). Dynamic systems of development: Change between complexity and chaos. New York: Harvester wheatsheaf. https://psycnet.apa.org/record/ 1995-97670-000

van Geert, P., \& van Dijk, M. (2002). Focus on variability: New tools to study intraindividual variability in developmental data. Infant Behavior $\& 3$ Development, 25 (4), 340-374. https://psycnet.apa.org/doi/10.10 16/S0163-6383(02)00140-6

Wellman, H. M. (2015). Making Minds: How theory of mind develops. Oxford University Press. https://doi.org/10.1093/acprof:oso/9 780199334919.001 .0001

Wellman, H. M., \& Woolley, J. (1990). From simple desires to ordinary beliefs: The early development of everyday psychology. Cognition, 35(3), 245-275. https://doi.org/1 0.1016/0010-0277(90)90024-E

Yan, Z., \& Fischer, K. (2002). Always under construction: Dynamic variations in adult cognitive microdevelopment. Human Development, 45(3), 141-160 https://doi.org $/ 10.1159 / 000057070$

Yott, J., \& Poulin-Dubois, D. (2016). Are infants' theory-of-mind abilities well integrated? Implicit understanding of intentions, desires, and beliefs. Journal of Cognition and Development, 17(5), 683-698. https://doi.or g/10.1080/15248372.2015.1086771

\section{Notas}

* Artículo de investigación 